\title{
Self-organized precipitation: an emerging method for preparation of unique polymer particles
}

\author{
Hiroshi Yabu \\ Polymer micro- and nano-particles have attracted much attention because of their promise for a variety of applications. This \\ focus review introduces an emerging method for producing nanostructured polymer particles, called self-organized precipitation \\ (SORP), and gives an overview of controlling the internal structure of polymer particles by using various polymer blends and \\ block-copolymer systems. The possible applications and future prospects of this technology are also discussed. \\ Polymer Journal (2013) 45, 261-268; doi:10.1038/pj.2012.151; published online 8 August 2012
}

Keywords: block copolymer; composite; particle; phase separation; polymer; polymer blend; self-organization

\section{INTRODUCTION}

Polymer micro- and nano-particles have attracted much attention because of their promise for a variety of applications, for example, filler for liquid column chromatography, ${ }^{1}$ drug delivery systems ${ }^{2}$ and templates for photonic crystals. ${ }^{3}$ These applications are based on the unique properties of polymer particles such as high surface/volume ratio and uniform size. The properties of polymer particles are generally governed by the following factors: constituent material, surface chemistry, size and shape. Through the study of colloidal dispersions of polymer particles, the first three of these factors have been thoroughly investigated. ${ }^{4}$ As the constituent polymer material governs various physical properties of particles, including thermal, mechanical and electrical properties, the polymer material should be chosen to suit the target application. Surface chemistry is also important from the point of view of polymer particle dispersibility. Polymer particles are used in the colloidal dispersions in various liquid media. Ionic moieties on the surface prevent particles from aggregating in an aqueous medium, and hydrophobic moieties stabilize the particles in organic solvents. The controlled assembly of particles can also be accomplished through ionic interactions between surface ionic moieties. ${ }^{5}$ Size is a critical parameter of polymer particles. For particles smaller than the capillary length of their medium, the effect of gravity is basically negligible, and their behavior is governed by Brownian motion and capillary force. The optical properties of polymer particles are also affected by their size, owing to Rayleigh scattering and Mie scattering. ${ }^{6}$

The shape factor is a relatively new topic in comparison with the other three factors. Put simply, this factor strongly depends on how the polymer particles are prepared. Particle shape has two aspects: external shape and internal structure. Conventional emulsion polymerization provides spherical particles only, because the droplets of template emulsion become spherical due to interfacial tension between monomer droplets and dispersion media. ${ }^{7}$ The recent development of seed polymerization allows for the production of various anisotropic particles having unique external morphologies. Particles with dumbbell-like ${ }^{8}$ and other unique morphologies 9 have been reported. However, few reports have examined controlling the internal structure of spherical polymer particles.

This focus review introduces an emerging method for producing nanostructured polymer particles, called self-organized precipitation (SORP), and gives an overview of controlling the internal structure of polymer particles by using various polymer blends and blockcopolymer systems. The possible applications and future prospects of this technology are also discussed.

\section{SELF-ORGANIZED PRECIPITATION}

A schematic illustration of the SORP method is shown in Figure $1 .^{10}$ Firstly, polymer is dissolved in a suitable good solvent. After mixing in a poor solvent, which is miscible with the good solvent, the good solvent is gradually evaporated. After complete exchange from the mixed solution to the poor solvent, polymer molecules precipitate as particles dispersed in the poor solvent. To selectively evaporate the good solvent, its boiling point should be lower than that of the poor solvent. After addition of the poor solvent, the solution should be handled gently and left to sit without stirring. Constant stirring leads to immediate bulk precipitation of polymer molecules. The SORP method has recently been reviewed. ${ }^{10}$

Particle size can be controlled by changing the preparation conditions including the solution concentration, the mixing ratio between the good and poor solvents, and the evaporation rate. ${ }^{11}$ Figure 2 shows a typical example of particle size control. In this case, high molecular weight polystyrene (PS) was dissolved in 
tetrahydrofuran, and water was used as the poor solvent. PS particles with size that can be tuned from $100 \mathrm{~nm}$ to micrometer scale were prepared by changing the concentration of PS and the mixing ratio between tetrahydrofuran and water.

The control of particle size is strongly related to the formation mechanism of polymer particles. The formation process of PS particle was observed by using the dynamic light scattering method. ${ }^{11}$ From

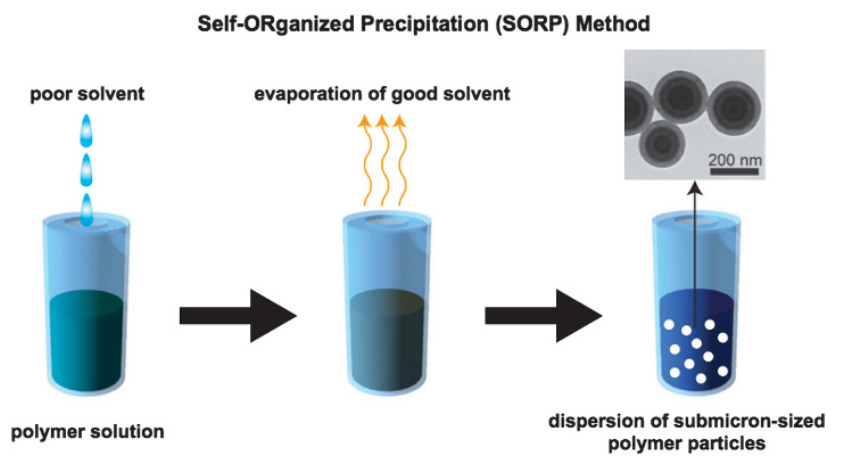

Figure 1 Schematic illustration of the SORP method. the dynamic light scattering measurement, the particle size gradually grew up with evaporation of a good solvent. In the good solvent, polymer molecules are basically in the random-coil state. After addition of the poor solvent, polymer molecules gradually come out of solution, and form the nuclei of the particles. The particles grow as other polymer molecules are adsorbed, until all the dissolved polymer molecules are consumed. The grown polymer particles spontaneously precipitate after complete evaporation of the good solvent. In this nucleation and growth process, there are two factors that determine the particle size: the number of nuclei and the number of molecules that do not form nuclei. Nucleation dominates particle formation when the mixed solution of good and poor solvents is supersaturated with polymer. When the solubility of the dissolved polymer is low or a large amount of poor solvent is added, the polymer molecules immediately precipitate, and an abundance of nuclei are formed. In such a case, most polymer molecules are consumed in nucleation and little growth follows, resulting in small particle size. On the other hand, the particles grow to micrometer scale when the mixed solution is not supersaturated. In such a case, fewer nuclei form and polymer is available for particle growth. Basically, size distribution of the formed particle is up to $10 \%$ with carefully controlling the preparation conditions such as solution temperature.
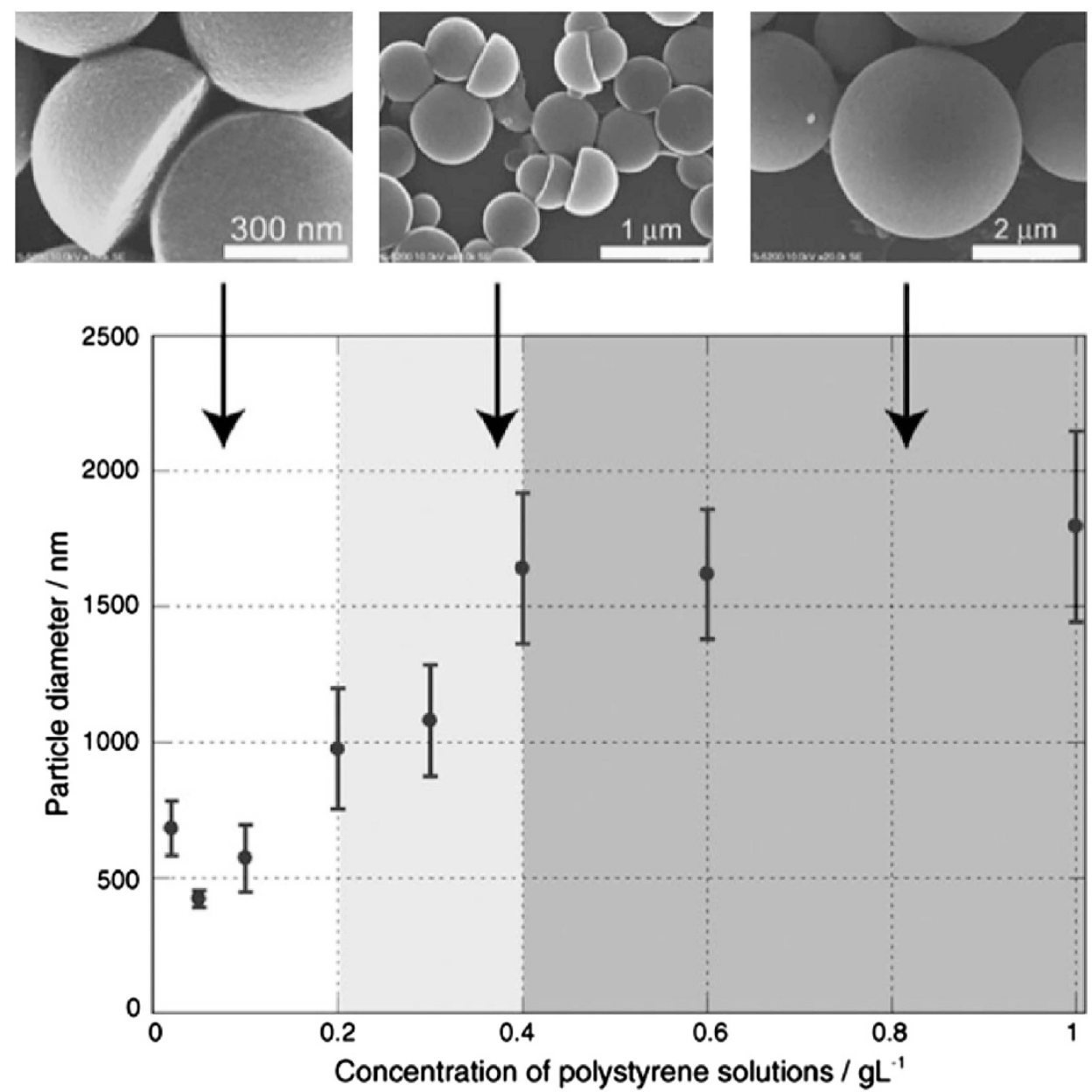

Figure 2 Relation between solution concentration and particle diameter. Scanning electron microscopy images show morphological changes from spherical to hemispherical with decreasing solution concentration. In this experiment, polystyrene (number average molecular weight (Mn) $=105.3 \mathrm{~kg}$ mol-1, weight average molecular weight $(\mathrm{Mw})=110.3 \mathrm{~kg} \mathrm{~mol}^{-1}, \mathrm{Mw} / \mathrm{Mn}=1.05$, Polymer Source Inc., Montreal, Quebec, Canada) was used for particle preparation. 
The preparation conditions affect the particle shape. When the concentration of the solution is low enough for nucleation to occur at the air/solution interface, the hemispherical particles are formed. ${ }^{12}$ At the early stage of good solvent evaporation, nucleation is limited to the air/solution interface in the case of low-concentration conditions. Owing to the formation of particles at only the air/solution interface, the particles grow anisotropically, and hemispherical particles are formed. After several hours of growth, evaporative cooling induces convection flows at the solution surface and a homogeneous dispersion of hemispherical particles is obtained.

Various polymers can be used in the preparation of particles by SORP. The principal criterion is that the polymer has suitable good and poor solvents. We have reported the use of SORP to prepare particles from commodity polymers, fluorescent polymers, biodegradable polymers and other polymer types. ${ }^{13}$ Serizawa et al. ${ }^{14}$ have reported preparation and size control of stereoregular poly(methyl methacrylate)s (PMMAs). Infrared (IR) measurements revealed that isotactic and syndiotactic PMMA particles had helical internal structures, whereas atactic PMMA particles were amorphous. Zhu et al. ${ }^{15}$ have also reported the preparation of monodisperse poly(DL-lactic acid) and poly(lactic-co-glycolic acid) particles (Figure 3).

The SORP method has also been used to prepare functional polymer particles. Matsukuma et al. ${ }^{16}$ have shown the preparation of fluorinated polymer particles, which exhibit high water repellency. The water-repellent microparticles can be used as stabilizers for various types of liquid marbles. Dye-containing polymer particles prepared by SORP can be used in optical nanochemosensors because of their high surface/volume ratio. Yu et al. ${ }^{17}$ have prepared particles from azobenzene-containing polymers, as well as assemblies of the particles. By using an ionic liquid, which is a molten salt with no vapor pressure, high thermal stability and high miscibility with water, particles of poly( $p$-xylene tetrahydrothiophenium chloride) were prepared from aqueous solution. ${ }^{18}$ After thermal treatment over $200{ }^{\circ} \mathrm{C}$, the particles were converted to poly ( $p$-phenylene vinylene) particles, which are fluorescent and conductive. Polyimide particles were also prepared from a polyamic acid precursor by the same procedure.

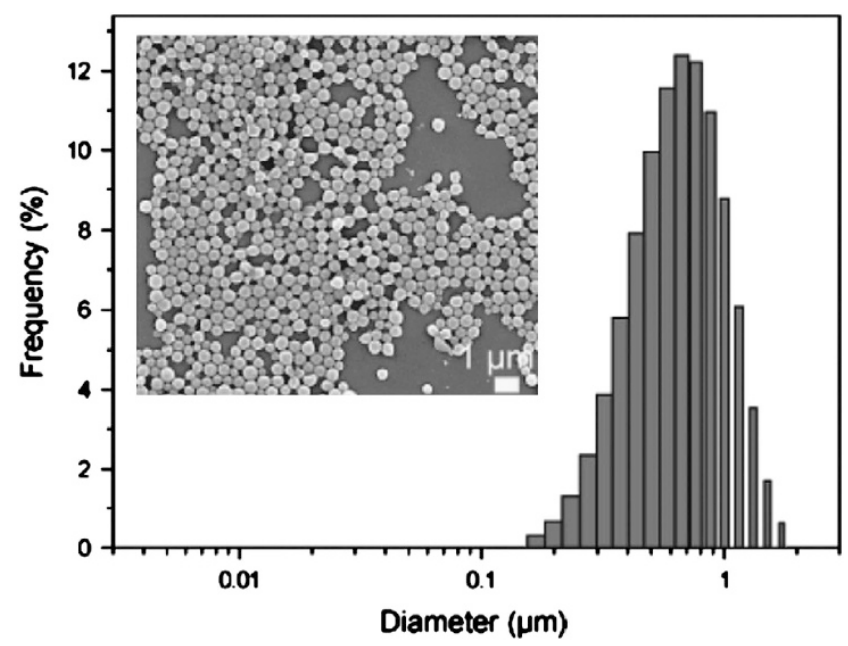

Figure 3 Scanning electron micrograph and histogram of particle diameters of poly(DL-lactic acid) particles (reprinted with permission from Liang et al. ${ }^{15}$ ). A full color version of this figure is available at Polymer Journal online.

\section{NANOSTRUCTURED PARTICLES}

The use of polymer blends, alloys or composites is a useful strategy for controlling the internal structure of polymer materials, and their mechanical, thermal and other physical properties. ${ }^{19}$ Microscale structures formed by phase separation between polymers and other materials provide unique physical properties that have not been obtained from polymer alone. Precipitation-based preparation methods of polymer particles can be applied to a wide variety of polymer materials.

\section{Polymer blend particles}

By using the SORP method, various kinds of polymer blend particles have been prepared. Hemispherical poly(1,4-isoprene) (PI) and PMMA particles have been prepared from dilute tetrahydrofuran solution of PI and PMMA. ${ }^{20}$ The Flory-Huggins solubility parameters of polymers strongly affect the internal structures in the polymer blend particles ${ }^{21}$ (Figure 4). When the solubility parameters of polymers are much different (that is, when their polarity is different), core-shell-structured particles are selectively obtained when water is used as the poor solvent. For example, PI/PMMA and PI/polyvinyl acetate systems form particles with a core-shell structure having a PI core. A PI/polyisobutylene system provides core-shell particles with a PI shell. In contrast, PI/PS and PI/poly(tert-butyl methacrylate) particles exhibit a Janus structure.

By using the relation between polymer polarity and particle structure, the dynamic control of the internal structure in polymer blend particles has been demonstrated by using stimuli-responsive polymer. When particles of PI and amphiphilic copolymer containing poly( $\mathrm{N}$-isopropyl acrylamide) were prepared by SORP in tetrahydrofuran/water, two types of internal structures (Janus and core-shell) were formed depending on preparation temperature. ${ }^{22}$

\section{Block-copolymer particles}

In 1999, there was one report about the preparation of nanostructured silica particles based on the self-assembly of block copolymers by atomization of a solution containing Pluronic 123, which is a wellknown water-soluble block copolymer, and silica source. ${ }^{23}$ A decade later, block-copolymer particles started to receive great interest owing to their potentials as platforms for various practical applications including drug delivery systems ${ }^{24}$ and so on. ${ }^{25}$

Amphiphilic block copolymers are known to form micelles of various shapes in a selective solvent. Many related works have been reviewed in the literature. ${ }^{26}$ Research on hydrophobic blockcopolymer particles, however, is limited due to difficulties in the preparation of hydrophobic block-copolymer particles. ${ }^{27}$ Block copolymers are difficult to prepare by conventional emulsion polymerization because their synthesis requires highly purified chemical agents and highly reactive initiators under rigorously controlled conditions in order to avoid chain transfer and termination processes. Recent development of living radical polymerization has enabled the preparation of block-copolymer particles by conventional emulsion polymerization. Ookubo et al. ${ }^{28}$ have reported that atom-transfer radical polymerization in miniemulsion provides block-copolymer particles with phaseseparation structures. Matyjaszewski reported that activators regenerated by electron transfer for atom-transfer radical polymerization) enabled living radical polymerization in emulsions under milder conditions in comparison with other living radical polymerization processes. ${ }^{29}$

The situation was drastically changed after the emergence of precipitation-based particle preparation processes using block 


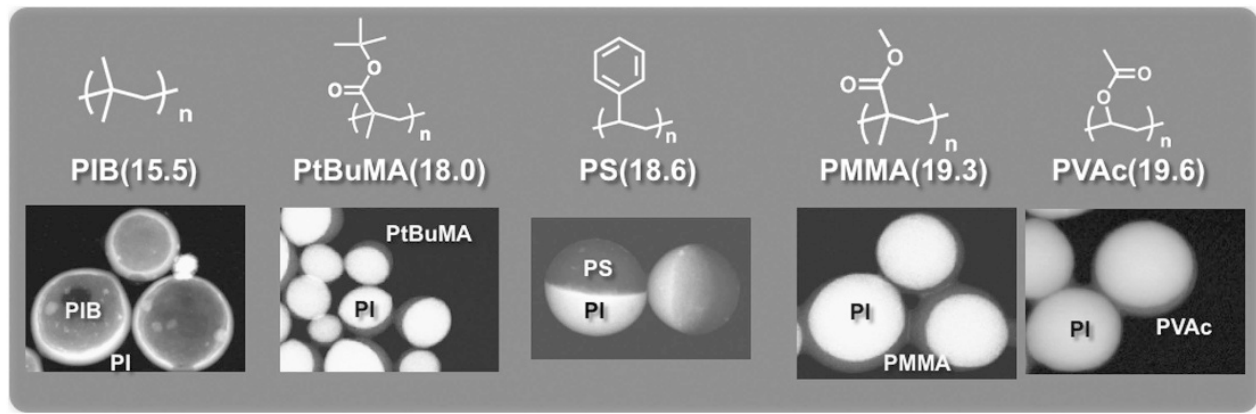

Figure 4 Scanning transmission electron micrographs of various polymer blend particles containing poly(1,4-isoprene) (white region). The numbers shown right sides of abbreviations of polymers mean solubility parameters of respective polymers. Whole polymers were purchased from Polymer Source, Inc. (poly(1,4-isoprene) (PI): molecular weight $(\mathrm{Mw})=12 \mathrm{kgmol}-1, \mathrm{Mw} / \mathrm{Mn}$ (molecular number) $=1.04$, polyisobutylene $(\mathrm{PIB})$ : $\mathrm{Mn}=19.5, \mathrm{Mw} / \mathrm{Mn}=1.4$, poly(tert-butyl methacrylate (PtBuMA): $17.5 \mathrm{~kg} \mathrm{~mol}^{-1}, \mathrm{Mw} / \mathrm{Mn}=1.04$, polystyrene $(\mathrm{PS}): \mathrm{Mn}=17 \mathrm{~kg} \mathrm{~mol}-1, \mathrm{Mw} / \mathrm{Mn}=1.03, \mathrm{poly}(\mathrm{methyl} \mathrm{methacrylate})$ (PMMA): $\mathrm{Mn}=15.8, \mathrm{Mw} / \mathrm{Mn}=1.06$, polyvinyl acetate (PVAc): $\mathrm{Mn}=41, \mathrm{Mw} / \mathrm{Mn}=1.5$ ).

copolymers. Through such processes, block-copolymer particles can be prepared without the use of sophisticated synthesis techniques. Following reports on the preparation of block-copolymer particles by precipitation-based particle formation techniques, increasing numbers of studies on block-copolymer particles have been reported. For example, PS-b-PI particles having unidirectionally stacked lamellae or onion-like phase-separated structures can be prepared by SORP. ${ }^{30}$ Structures with internal phase separation can be controlled by changing the copolymerization ratio, molecular weight and particle. Ho et al. ${ }^{31}$ reported that end-functionalized polymer blends form supramolecular assemblies similar to block copolymers in their particles and form unique microphase-separated structures. Particles from fluorine-containing block copolymers form various phaseseparated structures according to preparation conditions such as water content in the solution and preparation temperatures. ${ }^{32}$ Microphase-separated structures of ABC triblock copolymers in the particles were also investigated, as were more complicated structures with a variety of microphases due to the combination among three polymer moieties. ${ }^{33}$ Particles of block-copolymer blends or blockcopolymer/homopolymer blends show hierarchic microphaseseparated structures. ${ }^{34}$

We have also investigated block-copolymer/homopolymer blended particles formed by using the SORP method and determined the effect of homopolymer molecular weight. ${ }^{35}$ When the molecular weight of blended homopolymer is lower than the corresponding segment in the block copolymer, a morphological change occurred. On the other hand, the domain spacing or macrophase separation increases when the molecular weight of blended homopolymer is increased. Block-copolymer blends show more complex microphase separation in their particles.

Notably, the three-dimensional confinement effect on the phaseseparated structures of block copolymers have revealed by using such block-copolymer particles. Microphase separation of block copolymers in confined spaces was first investigated to solve engineering problems originating from defects in microdomains of microphaseseparated structures. ${ }^{36}$ Microphase-separated structures of block copolymers have been suggested as high-resolution nanoscale templates for patterned media-type memory devices ${ }^{37}$ and other semiconductor applications. ${ }^{38}$ Such templates would have resolution unobtainable by top-down microfabrication techniques. However, existence of defects prevents block copolymers from being used in practical semiconductor applications. Various types of surface topologies fabricated by top-down microfabrication techniques have been used for templates of microphase separation of block copolymers. This graphoepitaxy technique provides well-aligned and defect-free microphase-separated structures. ${ }^{39}$

Microphase-separated structures confined in limited dimensions exhibit structures different from their bulk states. Such exotic microphases were first investigated in thin films. ${ }^{40}$ Then, fibers prepared by electrospinning of block copolymers ${ }^{41}$ or molding block-copolymer melts in porous templates including porous anodic alumina ${ }^{42}$ were used as two-dimensional confinement fields. Extensive theoretical and experimental investigations have been done, and researchers in this field have found that helical structures, ${ }^{43}$ onedimensionally aligned cylinders and lamellae, and other unique microdomains ${ }^{44}$ can be formed in two-dimensional confinement spaces. There have been few reports on microphase-separated structures of block copolymers in three-dimensional confinement spaces, however.

Theoretical calculations for three-dimensionally confined microphase-separated structures have been studied by using self-consistent field theory. Li and co-workers or Shi and co-workers extensively investigated microphase-separated structures in spherically confined media by self-consistent field theory and Monte Carlo simulations, respectively, and proposed unique microphases. ${ }^{45-47}$ These theoretical studies indicated that the affinity of polymer segments in block copolymers for the matrix media $(a)$ and size ratio between the size of confined spaces (D) and domain spacing (L0) of microphase separation in the bulk state strongly affect three-dimensionally confined microphase separation.

There are two approaches to experimentally investigating threedimensional confinement effects; one is using three-dimensionally porous templates and the other is forming block-copolymer particles. Ozin, Manners, and co-workers have reported three-dimensionally confined microphase separation of block copolymers in colloidal crystals and inverse opal materials. ${ }^{48,49}$ Dichloromethane solution of lamella-forming poly(styrene- $b$-ferrocenylethylmethylsilane) was injected into the gap of the templates and dried to form confined microdomains of poly(styrene- $b$-ferrocenylethylmethylsilane). Small pores in inverse opal materials give spherical microdomains and large pores give onion-like microdomains. This inverse opal approach has been used to create organic-inorganic composites of block copolymers and hemispherical microdomains. ${ }^{50}$

In 2008, we reported the unique morphologies of PS-b-PI particles having low $D / L 0$ values. ${ }^{51}$ When the $D / L 0$ values greater than 2.0 , an onion-like phase was observed. On the other hand, in the case of 


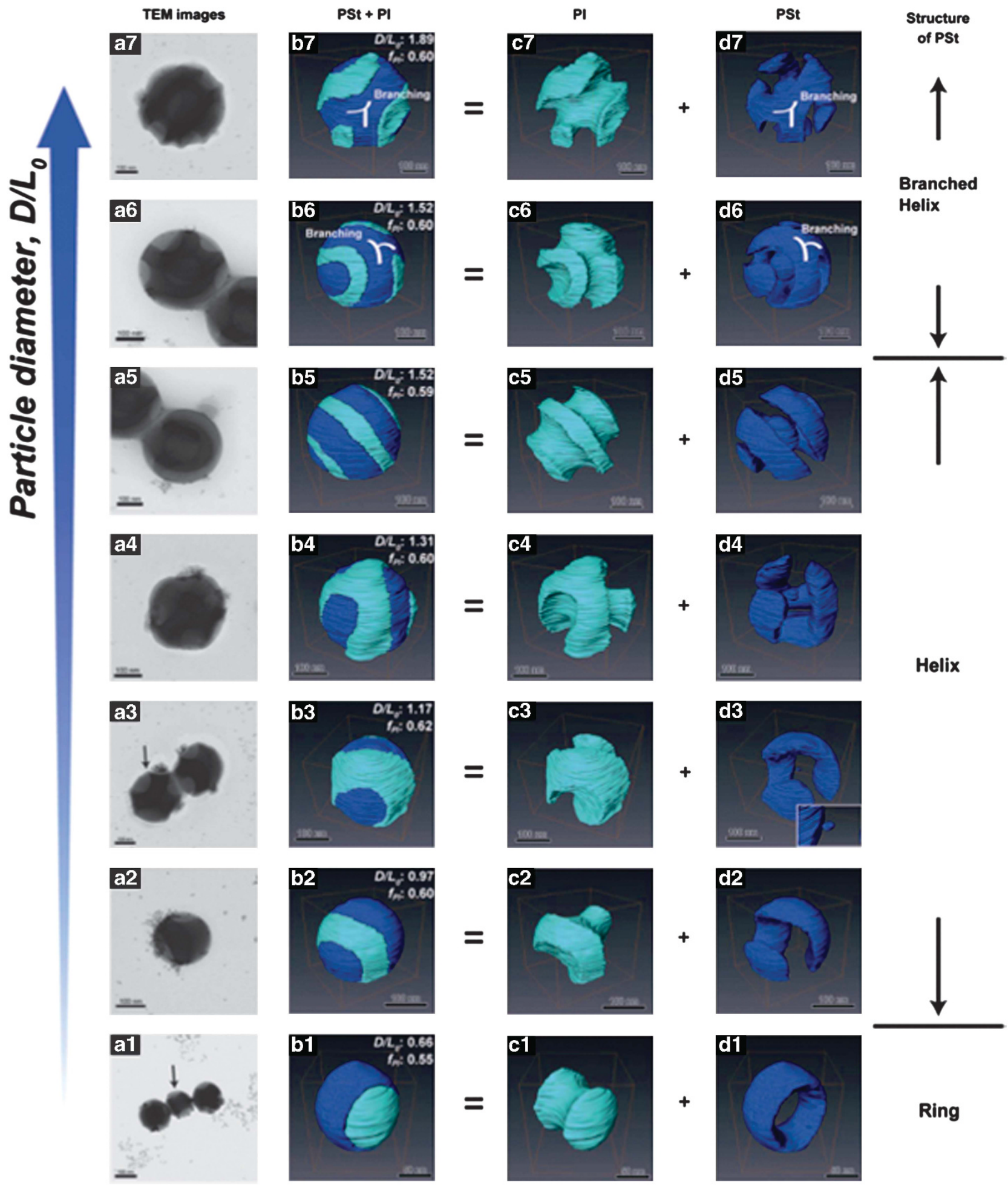

Figure 5 Transmission electron micrographs and three-dimensionally reconstructed images obtained by transmission electron microtomography measurement of various confined block-copolymer particles. When the particle size increased, corresponding to a high D/LO value, the morphology changed from annular to helical.

$1.0<D / L 0<2.0$, the unique morphologies including screw-, wheel-, and tennis ball-like structures have been observed. When the $D / \mathrm{L} 0$ values were lower than 1.0, a Janus structure was observed. These observations provided the first experimental evidence of three-dimensionally confined block-copolymer particles. The effects of solvent annealing on the confined block-copolymer particles were also investigated. ${ }^{52}$ Other unique phases were reported in composite particles of block copolymer and silica, particles of amphiphilic 

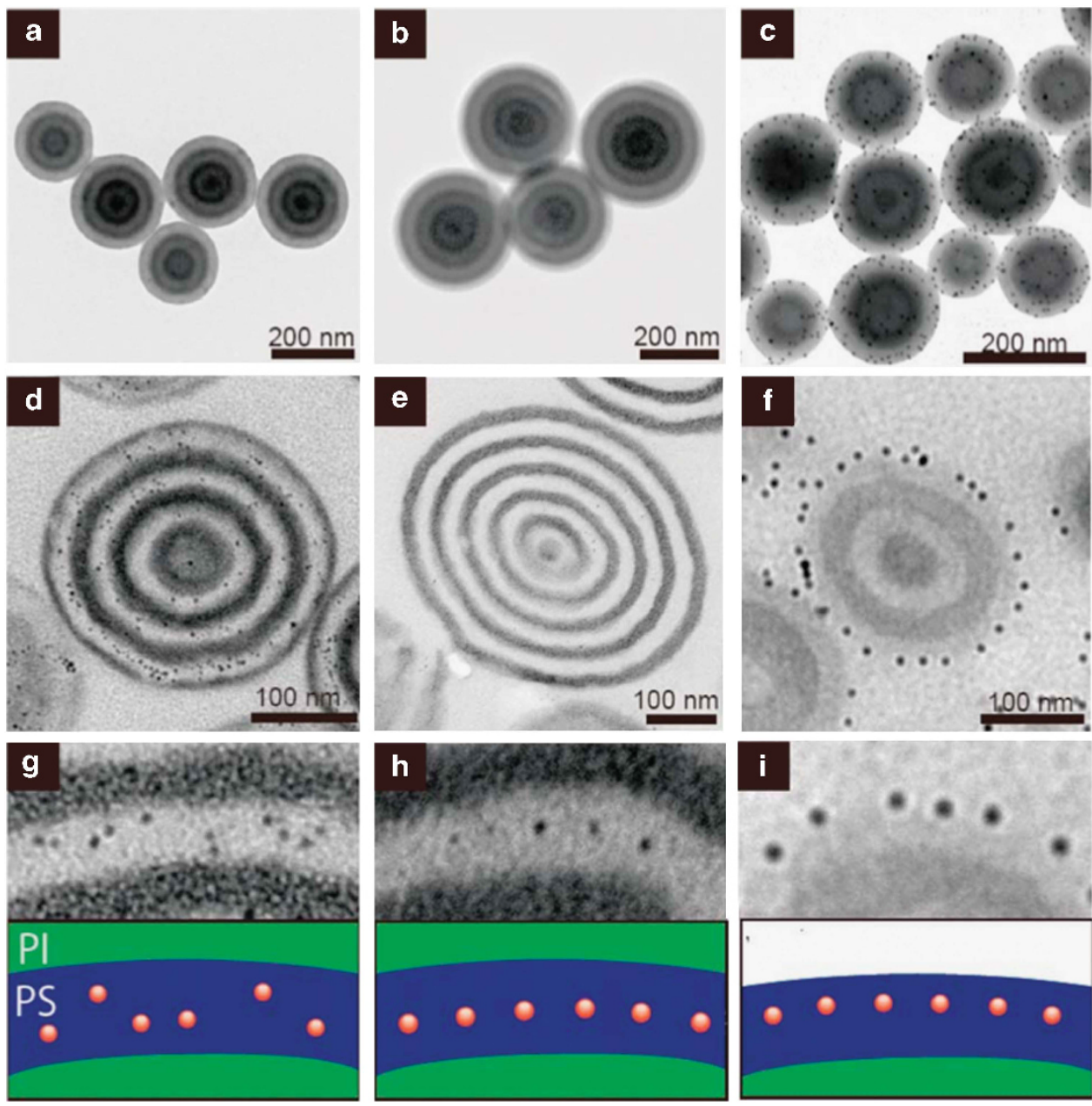

Figure 6 Composite particles of polystyrene-b-poly(1,4-isoprene) (PS-b-PI) and variously sized Au nanoparticles with polymer shells. Transmission electron microtomography (TEM) images of composite particles 1 (a) (PS-b-PI (molecular number $(\mathrm{Mn})_{(\mathrm{PS})}=45.0, \mathrm{Mn}_{(\mathrm{PI})}=31.0$, molecular weight $(\mathrm{Mw}) / \mathrm{Mn}=1.05$ Polymer Source Inc.) and thiol-terminated PS ( $\mathrm{Mw}=1.0 \mathrm{~kg} \mathrm{~mol}^{-1}, \mathrm{Mw} / \mathrm{Mn}=1.40$ )-stabilized Au nanoparticles (Au NPs, core diameter $\sim 3 \mathrm{~nm}$ )), 2 (b) (PSb-PI $\left(M_{(P S)}=45.0, M_{(P I)}=31.0, M w / M n=1.05\right.$, Polymer Source Inc.) and thiol-terminated PS (Mw = 11.5 kg mol $\left.{ }^{-1}, \mathrm{Mw} / \mathrm{Mn}=1.08\right)$-stabilized Au NPs), and 3 (c) $\left(P S-b-P I\left(M_{(P S)}=143.0, M_{(P I)}=81.0, M w / M n=1.05\right.\right.$, Polymer Source Inc.) and PS-b-P2VP $\left(M_{(P S)}=25.0, M_{(P 2 V P)}=23.5, M w / M n=1.05\right)-$ stabilized Au NPs (core diameter $\sim 10 \mathrm{~nm}$ )), cross-sectional TEM images of composite particles (d) 1 , (e) 2, and (f) 3 , close-up images of the crosssectional TEM images (g) 1, (h) 2, and (i) 3 are shown, respectively. Small PS-stabilized Au nanoparticles were selectively introduced into the PS phase, but large Au nanoparticles were localized at the particle surface.

double-comb diblock copolymers, and so forth. ${ }^{53}$ Most recently, detail microdomain structures in three-dimensionally confined spaces were revealed by a transmission electron microtomography technique ${ }^{54}$ (Figure 5). Three-dimensional observation of confined structures showed that deformation of microphase separation is selectively induced at the particle surface.

\section{Composite particles}

Through the introduction of inorganic materials into polymer particles, organic-inorganic composite particles having internal nanostructures and functions arising from the properties of inorganic materials can be realized. Toward this end, various polymer-stabilized inorganic nanoparticles have been used. Au nanoparticles ranging from a few nanometers to tens of nanometers can be synthesized by simple reduction of $\mathrm{HAuCl}_{4}$ in poly(styrene-b-vinylpyridine) (PS-bPVP) micelles dispersed in toluene. ${ }^{55}$ By this procedure, PS-b-PVPstabilized Au nanoparticles can be obtained. Composite particles of polymer blends and PS-b-PVP-stabilized Au nanoparticles can be prepared by SORP. ${ }^{56}$ As shown in section 'Polymer blend particles', Janus and core-shell phase-separated structures are formed in the particles depending on the combination of polymer sources. ${ }^{57}$ The internal phase-separated structures of the composite particles were basically the same as in the case of polymer blends, and $\mathrm{Au}$ nanoparticles were selectively introduced into the PS phase. cadmium sulfide (CdS) nanoparticles, which produce strong fluorescence emissions depending on their size, also synthesized in micelles of poly(styrene-b-acrylic acid) (PS-b-PAA) and also introduced into polymer blend particles. ${ }^{58}$ Silica particles with hydrophobic surface groups can also be assembled into nanostructured polymer particles by the SORP method..$^{59}$

In the case of composite particles of block copolymers and inorganic nanoparticles, the size ratios between the domain spacing of the single phase of block copolymer $(D)$ and the total diameter $(d)$ of inorganic nanoparticles including the polymer shells are important for selective introduction of nanoparticles (Figure 6). If $d \geqslant D$, the nanoparticles are not introduced into the phase-separated structures 
and are located on the surface of the particles. On the other hand, the particles are introduced into the polymer phase if $d<D .^{60}$

Electroless plating is another possible way to create organicinorganic composite particles. As poly(vinyl pyridine) (PVP) can form metal ion complexes, metal ions can be introduced into the PVP blocks in PS-b-PVP particles. After reduction of the metal ions, organic-inorganic composite particles can be obtained. ${ }^{61}$

From these examples, the inorganic nanoparticles can be introduced into targeted phases of polymer blend or block-copolymer particles by using stabilization of nanoparticles with polymers. As there are a wide variety of functional inorganic nanoparticles, various types of composite particles and unique material functions can be achieved by using the SORP method.

\section{APPLICATIONS AND FUTURE PROSPECTS}

Nanostructured polymer particles are expected to be applicable in biotechnology, optoelectronics and other practical fields depending on their nanostructures. Notable applications for each nanostructure are shown below.

Core-shell and multilayered particles composed of two or more different biodegradable polymers can be applied as carriers in drug delivery systems. If drugs are loaded into one or more phases of the particles, the drug will be slowly released. The core-shell and multilayered particles can be used as template for nanostructures. When metal nanoparticles are introduced into specific phases, the nanoparticles can be aligned along to the phase-separated structures. Such metallodielectric nanostructures resembling split ring resonators could be applicable to metamaterials, which have abnormal refractive indices. Core-shell-structured particles made of stimuli-responsive materials could be used as capsules that release functional materials under an external stimulus.

Organic-inorganic composite Janus particles containing metal nanoparticles and pigment nanoparticles are ideal pigments for electronic paper. Janus-type phase-separated structure might also be good light scattering materials due to their anisotropy.

Particles from block-copolymer or block-copolymer blend particles with cylindrical or spherical domains can be used as 'suprapolymer' structures, which is a hierarchic assembly of block copolymers. ${ }^{62}$ Nanowires, nanorings and nanodots have been created from phaseseparated block-copolymer or block-copolymer blend particles. These suprapolymers can be used as nanoscale electronic connectors and ring resonators.

1 Jacoby, M. Chromatography in the extreme. C\&EN. 86, 17-23 (2008).

2 Nasongkla, N., Bey, E., Ren, J., Ai, H., Khemtong, C., Guthi, J. S., Chin, S. -F., Sherry, A. D., Boothman, D. A. \& Gao, J. Multifunctional polymeric micelles as cancertargeted, MRI-ultrasensitive drug delivery systems. Nano Lett. 6, 2427-2430 (2006).

3 Zhang, J., Li, Y., Zhang, X. \& Yang, B. Colloidal self-assembly meets nanofabrication: from two-dimensional colloidal crystals to nanostructure arrays. Adv. Mater. 22, 4249-4269 (2010).

4 Caruso, F. Colloids and Colloid Assemblies (Wiley-VCH, Weinheim, 2004).

5 Wagner, C. S., Shehata, S., Henzler, K., Yuan, J. \& Wittemann, A. Towards nanoscale composite particles of dual complexity. J. Colloid and Interf. Sci. 355, 115-123 (2011).

6 Krieger, I. M. \& O'Neill, F. M. Diffraction of light by arrays of colloidal spheres. J. Am. Chem. Soc. 90, 3114-3120 (1968).

7 Landfester, K. The generation of nanoparticles in miniemulsions. Adv. Mater. 13 765-768 (2001).

8 He, J., Perez, M. T., Zhang, P., Liu, Y., Babu, T., Gong, J. \& Nie, Z. A general approach to synthesize asymmetric hybrid nanoparticles by interfacial reactions. J. Am. Chem. Soc. 134, 3639-3642 (2012).

9 Yang, M., Wang, G. \& Ma, H. An efficient approach for production of polystyrene/ poly(4-vinylpridine) particles with various morphologies based on dynamic control. Chem. Commun. 47, 911 (2010)
10 Yabu, H. Creation of functional and structured polymer particles by Self-Organized Precipitation (SORP). Bull. Chem. Soc. Jpn 85, 265-274 (2012).

11 Yabu, H., Higuchi, T., Ijiro, K. \& Shimomura, M. Spontaneous formation of polyme nanoparticles by good-solvent evaporation as a nonequilibrium process. Chaos 15 , 047505 (2005).

12 Higuchi, T., Yabu, H. \& Shimomura, M. Simple preparation of hemispherical polystyrene particles. Colloids and Surf. A 284-285, 250-253 (2006).

13 Shimomura, M. \& Yabu, H. Fabrication of organic/inorganic hybrid meso-structures by self-organization. Int. J. Nanosci. 1, 673-676 (2002).

14 Zhang, K. \& Serizawa, T. Preparation and characterization of surfactant-free nanoparticles composed of stereoregular poly(methyl methacrylate)s. J. Nanosci. Nanotech. 9, 591-597 (2009).

15 Liang, R. \& Zhu, J. Monodisperse PLA/PLGA nanoparticle fabrication through a surfactant-free route. J. Controlled Release 152, e129-e131 (2011).

16 Matsukuma, D., Watanabe, H., Yamaguchi, H. \& Takahara, A. Preparation of lowsurface-energy poly[2-(perfluorooctyl)ethyl acrylate] microparticles and its application to liquid marble formation. Langmuir 27, 1269-1274 (2011).

17 Liu, H., Kobayashi, T. \& Yu, H. Easy fabrication and morphology control of supramolecular liquid-crystalline polymer microparticles. Macromol. Rapid Commun. 32, 378-383 (2010).

18 Yabu, H., Tajima, A., Higuchi, T. \& Shimomura, M. Simple route for fabricating Poly(para-phenylene vinylene) (PPV) particles by using ionic liquids and a solvent evaporation process. Chem. Commun. 38, 4588-4589 (2008).

19 Shonaike, G. O. \& Simon, G. P. Polymer Blends and Alloys (Marcel Dekker Inc., New York, 1999)

20 Tajima, A., Higuchi, T., Yabu, H. \& Shimomura, M. Hemispherical polymer nanoparticles of poly(methyl methacrylate)-polyisoprene blend with core-shell structure. Colloids and Surf. A 313-314, 332-334 (2008).

21 Higuchi, T., Tajima, A., Yabu, H. \& Shimomura, M. Spontaneous formation of polymer nanoparticles with inner phase separation structures. Soft Matter 4, 1302-1305 (2008).

22 Motoyoshi, K., Tajima, A., Higuchi, T., Yabu, H. \& Shimomura, M. Static and dynamic control of phase separation structures in nanoparticles of polymer blends. Soft Matter 6, 1253-1257 (2010).

23 Lu, Y., Fan, H., Stump, A., Ward, T. L., Rieker, T. \& Brinker, C. J. Aerosol-assisted selfassembly of mesostructured spherical nanoparticles. Nature 398, 223-226 (1999).

24 Cenni, E., Granchi, D., Avnet, S., Fotia, C., Salerno, M., Micieli, D., Sarpietro, M. G., Pignatello, R., Castelli, F. \& Baldini, N. Biocompatibility of poly(d,l-lactideco-glycolide) nanoparticles conjugated with alendronate. Biomaterials 29, 1400-1411 (2008).

25 Wei, J., Wang, H., Deng, Y., Sun, Z., Shi, L., Tu, B., Luqman, M. \& Zhao, D. Solvent evaporation induced aggregating assembly approach to three-dimensional ordered mesoporous silica with ultralarge accessible mesopores. J. Am. Chem. Soc. 133, 20369-20377 (2011)

26 Hamley, I. W. Nanoshells and nanotubes from block copolymers. Soft Matter 1, 36 (2005).

27 Deng, R., Liu, S., Li, J., Liao, Y., Tao, J. \& Zhu, J. Mesoporous block copolymer nanoparticles with tailored structures by hydrogen-bonding-assisted self-assembly. Adv. Mater. 24, 1889-1893 (2012).

28 Kitayama, Y., Kagawa, Y., Minami, H. \& Okubo, M. Preparation of micrometer-sized, onion like multilayered block copolymer particles by two-step AGET ATRP in aqueous dispersed systems: effect of the second-step polymerization temperature. Langmuir 26, 7029-7034 (2010).

29 Matyjaszewski, K. Atom Transfer Radical Polymerization (ATRP): current status and future perspectives. Macromolecules 45, 4015-4428 (2012).

30 Yabu, H., Higuchi, T. \& Shimomura, M. Unique phase-separation structures of block - copolymer nanoparticles. Adv. Mater. 17, 2062-2065 (2005).

31 Huh, J., Jung, J. Y., Lee, J. U., Cho, H., Park, S., Park, C. \& Jo, W. H. Supramolecular assembly of end-functionalized polymer mixtures confined in nanospheres. ACS Nano 5, 115-122 (2011).

32 Qin, S., Li, H., Yuan, W. Z. \& Zhang, Y. Fluorine-containing block copolymer particles with surface and internal hierarchical microphase separation structures. Soft Matter $\mathbf{8}$, 2471-2476 (2012).

33 Hales, K., Chen, Z., Wooley, K. L. \& Pochan, D. J. Nanoparticles with tunable internal structure from triblock copolymers of PAA-b-PMA-b-PS. Nano Lett. 8, 2023-2026 (2008).

34 Yabu, H., Motoyoshi, K., Higuchi, T. \& Shimomura, M. Hierarchical structures in AB/AC type diblock-copolymer blend particles. Phys. Chem. Chem. Phys. 12, 11944 (2010).

35 Yabu, H., Sato, S., Higuchi, T., Jinnai, H. \& Shimomura, M. Creating suprapolymer assemblies: nanowires, nanorings, and nanospheres prepared from symmetric blockcopolymers confined in spherical particles. J. Mater. Chem. 22, 7672-7675 (2012).

36 Tada, Y., Akasaka, S., Yoshida, H., Hasegawa, H., Dobisz, E., Kercher, D. \& Takenaka, M. Directed self-assembly of diblock copolymer thin films on chemically-patterned substrates for defect-free nano-patterning. Macromolecules 41, 9267-9276 (2008).

37 Yang, X., Wan, L., Xiao, S., Xu, Y. \& Weller, D. K. Directed block copolymer assembly versuselectron beam lithography for Bit-patterned media with areal density of 1 terabit/ inch 2 and beyond. ACS Nano 3, 1844-1858 (2009).

$38 \mathrm{Kim}$, H. -C., Park, S. -M. \& Hinsberg, W. D. Block copolymer based nanostructures: materials, processes, and applications to electronics. Chem. Rev. 110, 146-177 (2010).

39 Cheng, J. Y., Ross, C. A., Smith, H. I. \& Thomas, E. L. Templated self-assembly of block copolymers: top-down helps bottom-up. Adv. Mater. 18, 2505-2521 (2006). 
40 Knoll, A., Horvat, A., Lyakhova, K., Krausch, G., Sevink, G., Zvelindovsky, A. \& Magerle, R. Phase behavior in thin films of cylinder-forming block copolymers. Phys. Rev. Lett. 89, 035501 (2002).

41 Stewart-Sloan, C. R. \& Thomas, E. L. Interplay of symmetries of block polymers and confining geometries. Eur. Polym. J. 47, 630-646 (2011).

42 Dobriyal, P., Xiang, H., Matsunaga, K., Chen, J. T., Jinnai, H. \& Russell, T. P. Cylindrically confined diblock copolymers. Macromolecules 42, 9082-9088 (2009).

43 Sevink, G. J. A. \& Zvelindovsky, A. V. Block copolymers confined in a nanopore: Pathfinding in a curving and frustrating flatland. J. Chem. Phys. 128, 084901 (2008).

44 Zhang, X., Harris, K. D., Wu, N. L. Y., Murphy, J. N. \& Buriak, J. M. Fast assembly of ordered block copolymer nanostructures through microwave annealing. ACS Nano 4, 7021-7029 (2010)

45 Feng, J. \& Ruckenstein, E. Self-assembling of ABC linear triblock copolymers in nanocylindrical tubes. J. Chem. Phys. 126, 124902 (2007).

46 Yu, B., Jin, Q., Ding, D., Li, B. \& Shi, A.-C. Confinement-induced morphologies of cylinder-forming asymmetric diblock copolymers. Macromolecules 41, 4042-4054 (2008).

47 Chi, P., Wang, Z., Li, B. \& Shi, A.-C. Soft confinement-induced morphologies of diblock copolymers. Langmuir 27, 11683-11689 (2011).

48 Arsenault, A. C., Rider, D. A., Tetrault, N., Chen, J. I. L., Coombs, N., Ozin, G. A. \& Manners, I. Block copolymers under periodic, strong three-dimensional confinement. J. Am. Chem. Soc. 127, 9954-9955 (2005).

49 Rider, D. A., Liu, K., Eloi, J. -C., Vanderark, L., Yang, L., Wang, J. -Y., Grozea, D., Lu, Z. -H., Russell, T. P. \& Manners, I. Controlling the morphologies of organometallic block copolymers in the 3-dimensional spatial confinement of colloidal and inverse colloidal crystals. Macromolecules 41, 2250-2259 (2008).

50 Yabu, H., Jinno, T., Koike, K., Higuchi, T. \& Shimomura, M. Three-dimensional assembly of gold nanoparticles in spherically confined microphase-separation structures of block-copolymers. Macromolecules 44, 5868-5873 (2011).

51 Higuchi, T., Tajima, A., Motoyoshi, K., Yabu, H. \& Shimomura, M. Frustrated phases of block copolymers in nanoparticles. Angew. Chem. Int. Ed. 47, 8044-8046 (2008).
52 Li, L., Matsunaga, K., Zhu, J., Higuchi, T., Yabu, H., Shimomura, M., Jinnai, H. Hayward, R. C. \& Russell, T. P. Solvent-driven evolution of block copolymer morphology under 3D confinement. Macromolecules 43, 7807-7812 (2010).

53 Pinna, M., Guo, X. \& Zvelindovsky, A. V. Block copolymer nanoshells. Polymer 49, 2797-2800 (2008).

54 Higuchi, T., Motoyoshi, K., Sugimori, H., Jinnai, H., Yabu, H. \& Shimomura, M. Threedimensional observation of confined phase-separated structures in block copolymer nanoparticles. Soft Matter 8, 3791-3797 (2012).

55 Möller, M., Spatz, J. P. \& Roescher, A. Gold nanoparticles in micellar poly (styrene)-bpoly (ethylene oxide) films - size and interparticle distance control in monoparticulate films. Adv. Mater. 8, 337-340 (1996).

56 Yabu, H., Koike, K., Motoyoshi, K., Higuchi, T. \& Shimomura, M. Preparation of coreshell organic-inorganic nanocomposite particles based on phase separation of a polymer blend. Chem. Lett. 38, 964-965 (2009).

57 Yabu, H., Koike, K., Motoyoshi, K., Higuchi, T. \& Shimomura, M. A novel route for fabricating metal-polymer composite nanoparticles with phase-separated structures. Macromol. Rapid Commun. 31, 1267-1271 (2010).

58 Yabu, H., Endo, A., Koike, K., Higuchi, T. \& Shimomura, M. Hierarchical assembly of CdS nanoparticles in polymer particles with phase separation structures. Polym. J. $\mathbf{4 3}$ 301-305 (2011).

59 Yabu, H. \& Sato, S. Controlled assembly of silica microspheres in spherically confined polymer particles by using self-organized precipitation (SORP) method. Colloid Polym. Sci. 291, 181-196 (2013).

60 Yabu, H., Koike, K., Higuchi, T. \& Shimomura, M. Nanoparticle arrangements in blockcopolymer particles having microphase separated structures. J. Polym. Sci. Part. B; Polym. Phys. 49, 1717-1722 (2011).

61 Yabu, H. Motoyoshi, K., Tajima, A., Higuchi, T \& Shimomura, M. Preparation and metallization of block-copolymer particles with phase separation structures. Kobunshi Ronbunshu 66, 438-441 (2009).

62 Higuchi, T., Tajima, A., Motoyoshi, K., Yabu, H. \& Shimomura, M. Suprapolymer structures from nanostructured polymer particles. Angew. Chem. Int. Ed. 48, 5125-5128 (2009).

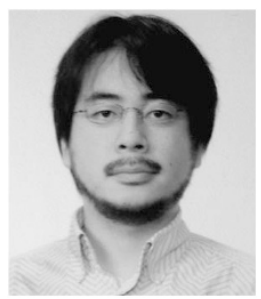

Hiroshi Yabu received $\mathrm{PhD}$ from Hokkaido University in 2004 under the supervision of Professor Masatsugu Shimomura (short-term graduation). He worked as an assistant professor of Nanotechnology Research Center, Research Institute for Electronic Science (RIES), Hokkaido University soon after his graduation. He also concurrently served as a visiting researcher of Frontier Research System, RIKEN Institute until 2007. From 2007, he worked as an assistant professor of Institute of Multidisciplinary Research for Advanced Materials (IMRAM), Tohoku University. He also concurrently served as a PRESTO Researcher, Japan Science and Technology Agency (JST) from 2008. He promoted an associate professor in 2010. His current scientific interests are organic-inorganic composite materials under confined spaces, self-organization and nano-structuring of functional materials. He received several awards including the CSJ Award for Young Chemists and the Award for Encouragement of Research in Polymer Science, the Society of Polymer Science, Japan in 2011. 